\title{
The Visualization of Spherical Patterns with Symmetries of the Wallpaper Group
}

\author{
Shihuan Liu $\mathbb{D}^{1,2}$ Ming Leng, ${ }^{1}$ and Peichang Ouyang $\mathbb{D}^{1}$ \\ ${ }^{1}$ School of Mathematics \& Physics, Jinggangshan University, Jian 343009, China \\ ${ }^{2}$ Sichuan Province Key Lab of Signal and Information Processing, Southwest Jiaotong University, Chengdu 611756, China \\ Correspondence should be addressed to Peichang Ouyang; g_fcayang@163.com
}

Received 17 October 2017; Accepted 1 January 2018; Published 12 February 2018

Academic Editor: Michele Scarpiniti

Copyright (c) 2018 Shihuan Liu et al. This is an open access article distributed under the Creative Commons Attribution License, which permits unrestricted use, distribution, and reproduction in any medium, provided the original work is properly cited.

\begin{abstract}
By constructing invariant mappings associated with wallpaper groups, this paper presents a simple and efficient method to generate colorful wallpaper patterns. Although the constructed mappings have simple form and only two parameters, combined with the color scheme of orbit trap algorithm, such mappings can create a great variety of aesthetic wallpaper patterns. The resulting wallpaper patterns are further projected by central projection onto the sphere. This creates the interesting spherical patterns that possess infinite symmetries in a finite space.
\end{abstract}

\section{Introduction}

Wallpaper groups (or plane crystallographic groups) are mathematical classification of two-dimensional repetitive patterns. The first systematic proof that there were only 17 possible wallpaper patterns was carried out by Fedorov in 1891 [1] and later derived independently by Pólya in 1924 [2]. Wallpaper groups are characterized by translations in two independent directions, which give rise to a lattice. Patterns with wallpaper symmetry can be widely found in architecture and decorative art [3-5]. It is surprising that the threedimensional 230 crystallographic groups were enumerated before the planar wallpaper groups.

The art of M. C. Escher features the rigorous mathematical structure and elegant artistic charm, which might be the one and only in the history of art. After his journey to the Alhambra, La Mezquita, and Cordoba, he created many mathematically inspired arts and became a master in creating wallpaper arts [6]. With the development of modern computers, there is considerable research on the automatic generation of wallpaper patterns. In [7], Field and Golubitsky first proposed the conception of equivariant mappings. They constructed equivariant mapping to generated chaotic cyclic, dihedral, and wallpaper attractors. Carter et al. developed an easier method that used equivariant truncated 2-dimensional
Fourier series to achieve it [8]. Chung and Chan [9] and $\mathrm{Lu}$ et al. [10] later presented similar ideas to create colorful wallpaper patterns. Recently, Douglas and John discovered a very simple approach to yield interesting wallpaper patterns of fractal characteristic [11].

The key idea behind [7-10] is equivariant mapping, which is not easy to achieve, since such mapping must be commutable with respect to symmetry group. In this paper, we present a simple invariant method to create wallpaper patterns. It has independent mapping form and only two parameters. Combined with the color scheme of orbit trap algorithm, our approach can be conveniently utilized to yield rich wallpaper patterns.

Escher's Circle Limits I-IV are unusual and visually attractive because they realized infinity in a finite unit disc. Inspired by his arts, we use central projection to project wallpaper patterns onto the finite sphere. This obtains the aesthetic patterns of infinite symmetry structure in the finite sphere space. Such patterns look beautiful. Combined with simulation and printing technologies, these computergenerated patterns could be utilized in wallpaper, textiles, ceramics, carpet, stained glass windows, and so on, producing both economic and aesthetic benefits.

The remainder of this paper is organized as follows. In Section 2, we first introduce some basic conceptions and the 
TABLE 1: The concrete invariant mapping $H_{f_{A}, B}(x)$ forms associated with 17 wallpaper groups. In the fourth column, the subscripts $A$ and $B$ identify the lattice kind ( $L_{s}$ represents square lattice, while $L_{d}$ represents diamond lattice) and wallpaper group type, respectively.

\begin{tabular}{|c|c|c|c|}
\hline $\begin{array}{l}\text { Wallpaper } \\
\text { group }\end{array}$ & $\begin{array}{l}\text { Point } \\
\text { group }\end{array}$ & Extra symmetry set & Invariant mapping \\
\hline$p 1$ & $C_{1}$ & None & $H_{f_{L_{s}, p 1}}(x)=\sum_{g \in C_{1}} f_{L_{s}}[g(x)]$ \\
\hline$p 2$ & $C_{2}$ & None & $H_{f_{L_{s}}, p_{2}}(x)=\sum_{g \in C_{2}} f_{L_{s}}[g(x)]$ \\
\hline pm & $D_{1}$ & $\sigma_{1}(a, b)=(a,-b)$ & $H_{f_{L_{s}}, p m}(x)=\sum_{g \in D_{1}} f_{L_{s}}[g(x)]+\sum_{g \in D_{1}} f_{L_{s}}\left[\left(\sigma_{1} g\right)(x)\right]$ \\
\hline pmm & $D_{2}$ & $\begin{array}{l}\sigma_{1}(a, b)=(a,-b) \\
\sigma_{2}(a, b)=(-a, b)\end{array}$ & $H_{f_{L_{s}, p m m}}(x)=\sum_{g \in D_{2}} f_{L_{s}}[g(x)]+\sum_{i=1}^{2}\left\{\sum_{g \in D_{2}} f_{L_{s}}\left[\left(\sigma_{i} g\right)(x)\right]\right.$ \\
\hline$p g$ & $D_{1}$ & $\sigma_{1}(a, b)=(\pi+a,-b)$ & $H_{f_{L_{s}, p g}}(x)=\sum_{g \in D_{1}} f_{L_{s}}[g(x)]+\sum_{g \in D_{1}} f_{L_{s}}\left[\left(\sigma_{1} g\right)(x)\right]$ \\
\hline pmg & $D_{2}$ & $\begin{array}{c}\sigma_{1}(a, b)=(\pi+a,-b) \\
\sigma_{2}(a, b)=(-a, b)\end{array}$ & $H_{f_{L_{s}}, p m g}(x)=\sum_{g \in D_{2}} f_{L_{s}}[g(x)]+\sum_{i=1}^{2}\left\{\sum_{g \in D_{2}} f_{L_{s}}\left[\left(\sigma_{i} g\right)(x)\right]\right.$ \\
\hline $\mathrm{Cm}$ & $D_{1}$ & $\begin{array}{c}\sigma_{1}(a, b)=(a,-b) \\
\sigma_{2}(a, b)=(\pi+a, \pi-b)\end{array}$ & $H_{f_{L_{s}}, c m}(x)=\sum_{g \in D_{1}} f_{L_{s}}[g(x)]+\sum_{i=1}^{2}\left\{\sum_{g \in D_{1}} f_{L_{s}}\left[\left(\sigma_{i} g\right)(x)\right]\right\}$ \\
\hline $\mathrm{Cmm}$ & $D_{2}$ & $\begin{array}{c}\sigma_{1}(a, b)=(a,-b) \\
\sigma_{2}(a, b)=(\pi-a, \pi+b) \\
\sigma_{3}(a, b)=(\pi+a, \pi-b) \\
\sigma_{4}(a, b)=(-a, b)\end{array}$ & $H_{f_{L_{s}}, c m m}(x)=\sum_{g \in D_{2}} f_{L_{s}}[g(x)]+\sum_{i=1}^{4}\left\{\sum_{g \in D_{2}} f_{L_{s}}\left[\left(\sigma_{i} g\right)(x)\right]\right.$ \\
\hline$p 4$ & $\mathrm{C}_{4}$ & None & $H_{f_{L_{s}}, p 4}(x)=\sum_{g \in C_{4}} f_{L_{s}}[g(x)]$ \\
\hline$p 4 g$ & $D_{4}$ & $\sigma_{1}(a, b)=(\pi+a,-b)$ & $H_{f_{L_{s}, p 4 g}}(x)=\sum_{g \in D_{4}} f_{L_{s}}[g(x)]+\sum_{g \in D_{4}} f_{L_{s}}\left[\left(\sigma_{1} g\right)(x)\right]$ \\
\hline$p 4 m$ & $D_{4}$ & $\sigma_{1}(a, b)=(a,-b)$ & $H_{f_{L_{s}}, p 4 m}(x)=\sum_{g \in D_{4}} f_{L_{s}}[g(x)]+\sum_{g \in D_{4}} f_{L_{d s}}\left[\left(\sigma_{1} g\right)(x)\right]$ \\
\hline$p g g$ & $D_{2}$ & $\begin{array}{l}\sigma_{1}(a, b)=(\pi+a, \pi-b) \\
\sigma_{2}(a, b)=(\pi-a, \pi+b)\end{array}$ & $H_{f_{L_{s}, p g g}}(x)=\sum_{g \in D_{2}} f_{L_{s}}[g(x)]+\sum_{i=1}^{2}\left\{\sum_{g \in D_{2}} f_{L_{s}}\left[\left(\sigma_{i} g\right)(x)\right]\right.$ \\
\hline p3 & $C_{3}$ & None & $H_{f_{L_{d}}, p^{3}}(x)=\sum_{g \in C_{3}} f_{L_{d}}[g(x)]$ \\
\hline$p 3 m 1$ & $D_{3}$ & $\sigma_{1}(a, b)=(-a, b)$ & $H_{f_{L_{d}}, p 3 m 1}(x)=\sum_{g \in D_{3}} f_{L_{d}}[g(x)]+\sum_{g \in D_{3}} f_{L_{d}}\left[\left(\sigma_{1} g\right)(x)\right]$ \\
\hline$p 31 m$ & $D_{3}$ & $\sigma_{1}(a, b)=(a,-b)$ & $H_{f_{L_{d}}, p 31 m}(x)=\sum_{g \in D_{3}} f_{L_{d}}[g(x)]+\sum_{g \in D_{3}} f_{L_{d}}\left[\left(\sigma_{1} g\right)(x)\right]$ \\
\hline$p 6$ & $C_{6}$ & None & $H_{f_{L_{d}}, p 6}(x)=\sum_{g \in C_{6}} f_{L_{d}}[g(x)]$ \\
\hline$p 6 m$ & $D_{6}$ & $\sigma_{1}(a, b)=(a,-b)$ & $H_{f_{L_{d}} p \sigma m}(x)=\sum_{g \in D_{6}} f_{L_{d}}[g(x)]+\sum_{g \in D_{6}} f_{L_{d}}\left[\left(\sigma_{1} g\right)(x)\right]$ \\
\hline
\end{tabular}

lattices with respect to wallpaper groups. To create patterns with symmetries of the wallpaper group, we will explicitly construct invariant mappings associated with 17 wallpaper groups (the concrete mapping forms are summarized in Table 1) in Section 3. In Section 4, we describe how to create colorful wallpaper patterns. Finally, we show some spherical wallpaper patterns obtained by central projection in Section 5.

\section{The Lattice of Wallpaper Groups}

In geometry and group theory, a lattice in 2-dimensional Euclidean plane $R^{2}$ is essentially a subgroup of $R^{2}$. Or, equivalently, for any basis vectors of $R^{2}$, the subgroup of all linear combinations with integer coefficients of the vectors forms a lattice $[12,13]$. Since a lattice is a finitely generated free abelian group, it is isomorphic to $Z^{2}$ and fully spans the real vector space $R^{2}$ [14]. A lattice may be viewed as a regular tiling of a space by a primitive cell. Lattices have many significant applications in pure mathematics, particularly in connection to Lie algebras, number theory, and group theory [15].

In this section, we mainly introduce the lattices associated with wallpaper group. Firstly, we introduce some basic conceptions.

The symmetry group of an object is the set of all isometries under which the object is invariant with composition as the group operation. A point group (sometimes called rosette group) is a group of isometries that keep at least one point fixed.

Point groups in $R^{2}$ come in two infinite families: dihedral group $D_{n}$ which is the symmetry group of a regular polygon 
and cyclic group $C_{n}$ that only comprises rotation transformations of $D_{n}$. Let $R_{n}=\left(\begin{array}{cc}\cos (2 \pi / n) & -\sin (2 \pi / n) \\ \sin (2 \pi / n) & \cos (2 \pi / n)\end{array}\right)$ and $T=\left(\begin{array}{cc}-1 & 0 \\ 0 & 1\end{array}\right)$. Then their matrix group can be represented as $C_{n}=\left\{R_{n}^{i}, i=\right.$ $1,2,3, \ldots, n\}$ and $D_{n}=C_{n} \cup\left\{T R_{n}^{i}, i=1,2,3, \ldots, n\right\}$.

A wallpaper group is a type of topologically discrete group in $R^{2}$ which contains two linearly independent translations. A lattice in $R^{2}$ is the symmetry group of discrete translational symmetry in two independent directions. A tiling with this lattice of translational symmetry cannot have more but may have less symmetry than the lattice itself. Let $L$ be a lattice in $R^{2}$. A lattice $L^{*}$ is called the dual lattice of $L$ if, $\forall u \in L$ and $\forall v \in L^{*}$, the inner product $u \cdot v$ is an integer, where $u$ and $v$ are vectors in $R^{2}$. Let $M$ be a mapping from $R^{2}$ to $R^{2}$ and let $G$ be a symmetry group in $R^{2} ; M$ is called an invariant mapping with respect to $G$ if, $\forall x \in R^{2}$ and $\forall g \in G, M(x)=M(g x)$.

By the crystallographic restriction theorem, there are only 5 lattice types in $R^{2}$ [16]. Although wallpaper groups have totally 17 types, their lattices can be simplified into two lattices: square and diamond lattices. For convenience, we require that the inner product of the mutual dual lattice of a wallpaper group be an integer multiple of $2 \pi$. Throughout the paper, for square lattice, we choose lattice $L_{s}=\{(1,0),(0,1)\}$ with dual lattice $L_{s}^{*}=\{2 \pi(1,0), 2 \pi(0,1)\}$; for diamond lattice, we choose lattice $L_{d}=\{(1,0),(1 / 2)(-1, \sqrt{3})\}$ with dual lattice $L_{d}^{*}=\{(2 \pi / \sqrt{3})(\sqrt{3},-1), 2 \pi(0,-2 / \sqrt{3})\}$.

In this paper, we use standard crystallographic notations of wallpaper groups $[16,17]$. Among 17 wallpaper groups, $p 1$, p2, pm, pmm, pg, pmg, cm, cmm, p4,pgg, p4g, and p4m possess square lattice, while $p 3, p 3 m 1, p 31 m, p 6$, and $p 6 m$ possess diamond lattice.

\section{Invariant Mapping with respect to Wallpaper Groups}

In this section, we explicitly construct invariant mappings associated with wallpaper groups. To this end, we first prove the following lemma.

Lemma 1. Suppose that $f_{i}(i=1,2,3,4)$ are sine or cosine functions, $G$ is a wallpaper group with lattice $L=\{A, B\}, L^{*}=$ $\left\{A^{*}, B^{*}\right\}$ is the dual lattice of $L$, and $a$ and $b$ are real numbers. Then mapping

$$
f_{L}(x)=\left(\begin{array}{c}
a f_{1}\left\{\sum_{v \in L} f_{2}(x \cdot v)+\sum_{v \in L}(x \cdot v)\right\} \\
b f_{3}\left\{\sum_{v \in L} f_{4}(x \cdot v)+\sum_{v \in L}(x \cdot v)\right\}
\end{array}\right),
$$

$\forall x \in R^{2}$,

is invariant with respect to $L^{*}$, or $f_{L}(x)$ has translation invariance of $L^{*}$; that is,

$$
\left(\begin{array}{l}
a f_{1}\left\{\sum_{v \in L} f_{2}((u+x) \cdot v)+\sum_{v \in L}((u+x) \cdot v)\right\} \\
b f_{3}\left\{\sum_{v \in L} f_{4}((u+x) \cdot v)+\sum_{v \in L}((u+x) \cdot v)\right\}
\end{array}\right)
$$

$$
=\left(\begin{array}{l}
a f_{1}\left\{\sum_{v \in L} f_{2}(x \cdot v)+\sum_{v \in L}(x \cdot v)\right\} \\
b f_{3}\left\{\sum_{v \in L} f_{4}(x \cdot v)+\sum_{v \in L}(x \cdot v)\right\}
\end{array}\right)=f_{L}(x),
$$

where $u=m A^{*}+n B^{*}, m, n \in Z$.

Proof. Since $L^{*}$ is the dual lattice of $L, \forall v \in L$, we have $u \cdot v=$ $\left(m A^{*}+n B^{*}\right) \cdot v=m\left(A^{*} \cdot v\right)+n\left(B^{*} \cdot v\right)=2 k \pi$ for certain $k \in Z$. Thus we get $f_{i}\left\{\sum_{v \in L} f_{j}((u+x) \cdot v)+\sum_{v \in L}((u+x) \cdot v)\right\}=$ $f_{i}\left\{\sum_{v \in L} f_{j}(x \cdot v)+\sum_{v \in L}(x \cdot v)\right\}$, since $f_{i}$ and $f_{j}$ are functions of period $2 \pi(i, j=1,2,3,4)$. Consequently, the mapping $f_{L}(x)$ constructed by $f_{i}(i=1,2,3,4)$ satisfies (2). This completes the proof.

Essentially Lemma 1 says that $f_{L}(x)$ is a double period mapping (of period $2 \pi$ ) along the independent translational directions of $L^{*}$.

Theorem 2. Let $G$ be a finite group realized by $2 \times 2$ matrices acting on $R^{2}$ by multiplication on the right and let $f$ be an arbitrary mapping from $R^{2}$ to $R^{2}$. Then mapping

$$
H_{f, G}(x)=\sum_{g \in G} f[g(x)], \quad x \in R^{2},
$$

is an invariant mapping with respect to $G$.

Proof. For $\sigma \in G$, by closure of the group operation, we see that $g \sigma$ runs through $G$ as $g$ does. Therefore we have

$$
\begin{aligned}
H_{f, G}[\sigma(x)] & =\sum_{g \in G} f[\sigma(g x)]=\sum_{g^{*} \in G} f\left[g^{*}(x)\right] \\
& =H_{f, G}(x),
\end{aligned}
$$

where $g^{*}=\sigma g \in G$. This means that $H_{f, G}(x)$ is an invariant mapping with respect to $G$.

Combining Lemma 1 and Theorem 2, we immediately derive the following theorem.

Theorem 3. Let $f$ in Theorem 2 have the form $f_{L}$ as in Lemma 1. Suppose that $G$ is a cyclic group $C_{n}$ or dihedral group $D_{n}$ with lattice $L ; L^{*}$ is the dual lattice associated with L. Assume that $H_{f_{L}, G}(x)$ is a mapping from $R^{2}$ to $R^{2}$ of the following form:

$$
H_{f_{L}, G}(x)=\sum_{g \in G} f_{L}[g(x)], \quad x \in R^{2} .
$$

Then $H_{f_{L}, G}(x)$ is an invariant mapping with respect to both $G$ and $L^{*}$.

Wallpaper groups possess globally translation symmetry along two independent directions as well as locally point group symmetry. For the wallpaper groups that only have symmetries of a certain point group, mapping $H_{f_{L}, G}(x)$ in 


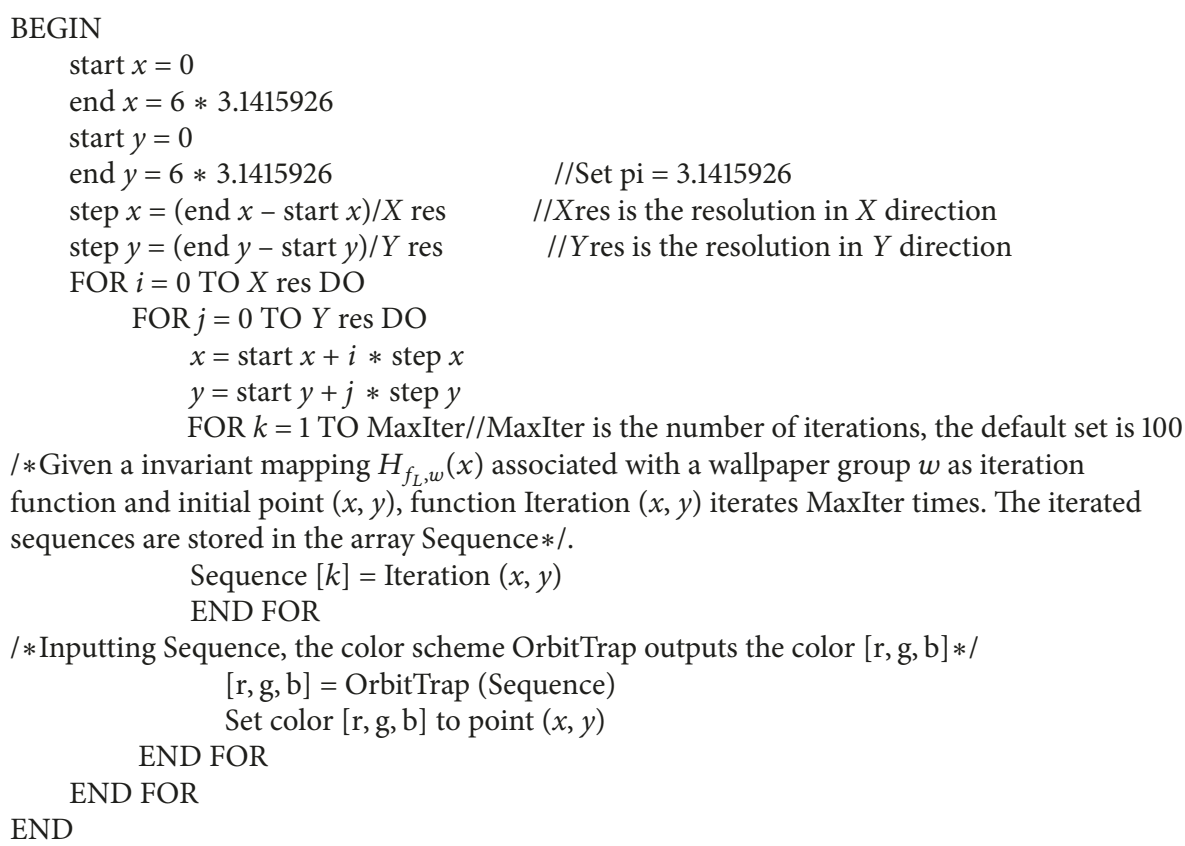

AlgoRIthm 1: CreatingWallpaperPattern()// algorithm for creating patterns with the wallpaper symmetry.

Theorem 3 can be used to create wallpaper patterns well. However, except for the symmetries of a point group, some wallpaper groups may possess other symmetries. For example, except for symmetries of dihedral group $D_{3}$, wallpaper group $p 31 \mathrm{~m}$ still has a reflection along horizontal direction, say symmetry $\sigma_{1}(a, b)=(a,-b)\left((a, b) \in R^{2}\right)$; besides $D_{2}$ symmetry, wallpaper group pmm contains perpendicular reflections; that is, $\sigma_{1}(a, b)=(a,-b)$ and $\sigma_{2}(a, b)=(-a, b)$.

For a wallpaper group $w$ with extra symmetry set $\Delta=$ $\left\{\sigma_{1}, \sigma_{2}, \sigma_{3}, \ldots, \sigma_{n}\right\}$, based on mapping (5), we add proper terms so that the resulting mapping $H_{f_{L}, w}(x)$ is also invariant with respect to $w$. This is summarized in Theorem 4 .

Theorem 4. Let $f$ in Theorem 2 have the form $f_{L}$ in Lemma 1. Suppose that $w$ is a wallpaper group with symmetry group $G$ and extra symmetry set $\Delta=\left\{\sigma_{1}, \sigma_{2}, \sigma_{3}, \ldots, \sigma_{n}\right\}$. Assume that $L$ is the lattice of $w$ and $L^{*}$ is the dual lattice associated with $L$. Let $H_{f_{L}, w}(x)$ be a mapping from $R^{2}$ to $R^{2}$ of the following form:

$$
\begin{aligned}
H_{f_{L}, w}(x)= & \sum_{g \in G} f_{L}[g(x)] \\
& +\sum_{i=1}^{n}\left\{\sum_{g \in G, \sigma_{i} \in \Delta} f_{L}\left[\left(\sigma_{i} g\right)(x)\right]\right\}, \quad x \in R^{2} .
\end{aligned}
$$

Then $H_{f_{L}, w}(x)$ is an invariant mapping with respect to both $w$ and $L^{*}$.

We refer the reader to $[7,8,17]$ for more detailed description about the extra symmetry set of wallpaper groups. By Theorems 3-4, we list the invariant mappings associated with 17 wallpaper groups in Table 1.

\section{Colorful Wallpaper Patterns from Invariant Mappings}

Invariant mapping method is a common approach used in creating symmetric patterns [18-23]. Color scheme is an algorithm that is used to determine the color of a point. Given a color scheme and domain $D$, by iterating invariant mapping $H_{f_{L}, w}(x), x \in D$, one can determine the color of $x$. Coloring points in $D$ pointwise, one can obtain a colorful pattern in $D$ with symmetries of the wallpaper group $w$. Figures 1-2 are four wallpaper patterns obtained in this manner. These patterns were created by $\mathrm{VC}++6.0$ on a PC (SVGA). In Algorithm 1, we provide the pseudocode so that the interested reader can create their own colorful wallpaper patterns.

The color scheme used in this paper is called orbit trap algorithm [10]. We refer the reader to [10, 20] for more details about the algorithm (the algorithm is named as function OrbitTrap() in Algorithm 1). It has many parameters to adjust color, which could enhance the visual appeal of patterns effectively. Compared with the complex equivariant mapping constructed in [7-10], our invariant mappings possess not only simple form but also sensitive dynamical system property, which can be used to produce infinite wallpaper patterns easily. For example, Figures 1(a) and 2(b) were created by mappings $H_{f_{L_{s}}, p 4 m}(x)$ and $H_{f_{L_{d}}, p 3 m 1}(x)$, respectively, in which the specific mappings $f_{L_{s}}$ and $f_{L_{d}}$ were

$$
f_{L_{s}}=\left(\begin{array}{c}
2.12 \cos \left\{\sum_{v \in L_{s}} \cos (x \cdot v)+\sum_{v \in L_{s}}(x \cdot v)\right\} \\
1.03 \cos \left\{\sum_{v \in L_{s}} \sin (x \cdot v)+\sum_{v \in L_{s}}(x \cdot v)\right\}
\end{array}\right),
$$




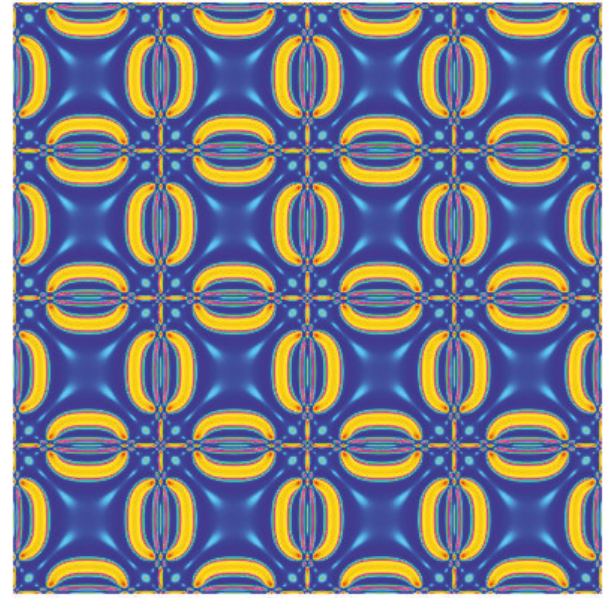

(a)

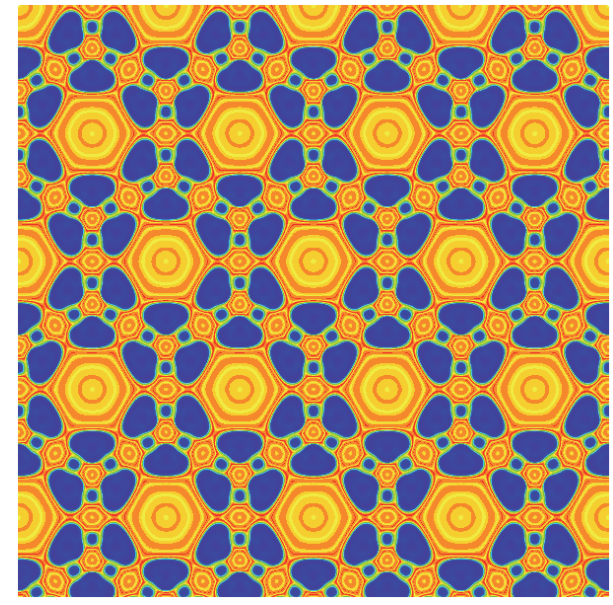

(b)

FIgURE 1: Colorful wallpaper patterns with the $p 4 m$ (a) and $p 6 m$ (b) symmetry.

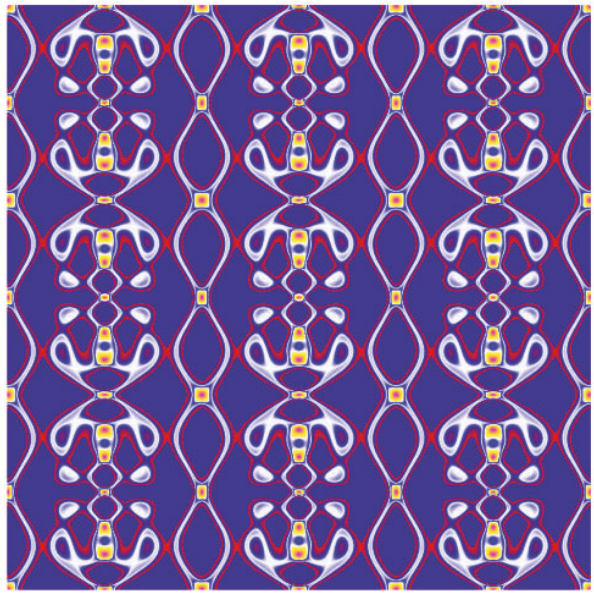

(a)

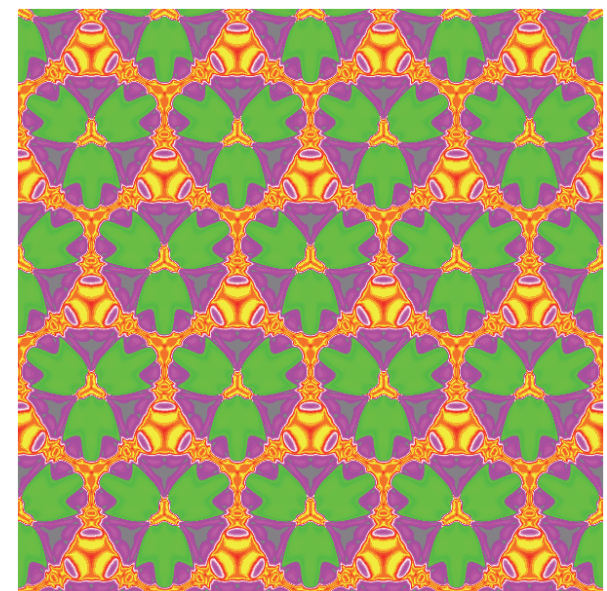

(b)

FIgURE 2: Colorful wallpaper patterns with the pmm (a) and $p 3 m 1$ (b) symmetry.

$$
f_{L_{d}}=\left(\begin{array}{c}
1.1 \cos \left\{\sum_{v \in L_{d}} \sin (x \cdot v)+\sum_{v \in L_{d}}(x \cdot v)\right\} \\
-0.52 \sin \left\{\sum_{v \in L_{d}} \cos (x \cdot v)+\sum_{v \in L_{d}}(x \cdot v)\right\}
\end{array}\right) .
$$

It seems that the deference between (7) and (8) is not very significant. However, by Table 1, mappings $H_{f_{L_{s}}, p 4 m}(x)$ and $H_{f_{L_{d}}, p 3 m 1}(x)$ have 16 and 12 summation terms, respectively. The cumulative difference will be very obvious, which is enough to produce different style patterns.

\section{Spherical Wallpaper Patterns by Central Projection}

In this section, we introduce central projection to yield spherical patterns of the wallpaper symmetry.
Let $S^{2}=\left\{(a, b, c) \in R^{3} \mid a^{2}+b^{2}+c^{2}=1\right\}$ be the unit sphere in $R^{3}$, let $Z=F$ be a projection plane, where $F$ is a negative constant. Assume that $P(a, b, c) \in R^{3}$; then $P(a, b, c) \in R^{3}$ and $P^{\prime}(-a,-b,-c) \in R^{3}$ are a pair of antipodal points. For any point $P(a, b, c) \in R^{3}$, there exist a unique line $L$ through the origin $(0,0,0)$ and $P$ (and $\left.P^{\prime}\right)$ which intersects the projection plane $Z=F$ at point $(\alpha, \beta, F)$. Denote the projection by $\tau$. By analytic geometry, it is easy to check that

$$
\left[\begin{array}{l}
\alpha \\
\beta \\
F
\end{array}\right]=\tau(a, b, c)=\tau(-a,-b,-c)=\left[\begin{array}{l}
a \\
b \\
c
\end{array}\right] \frac{F}{c} .
$$

Because the projection point is at the center of $S^{2}$, we call $\tau$ as central projection.

The choice of the plane $Z=F$ has a great influence on the spherical patterns. If plane $Z=F$ is too close to coordinate plane $X O Y$, the resulting spherical pattern only shows a few 


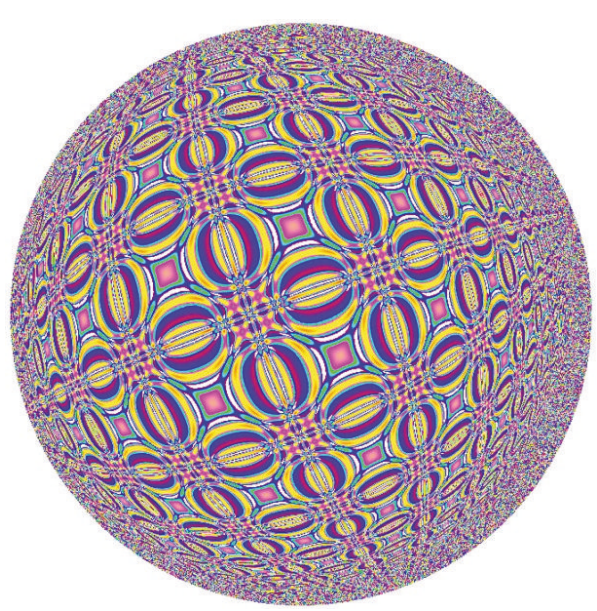

(a)

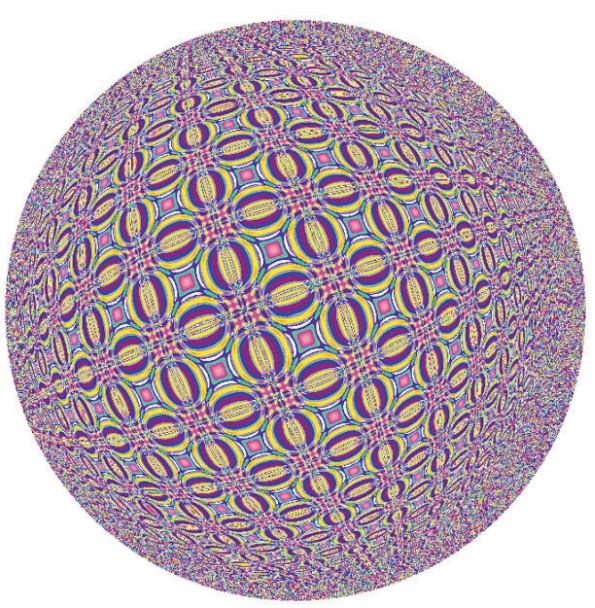

(b)

FIGURE 3: Two spherical wallpaper patterns with the $p 4 m$ symmetry, in which the projection plane was set as $Z=-2 \pi$ (a) and $Z=-4 \pi$ (b).

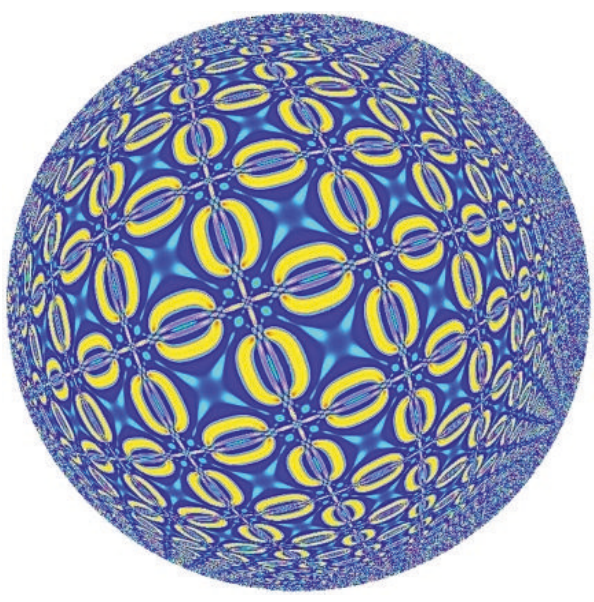

(a)

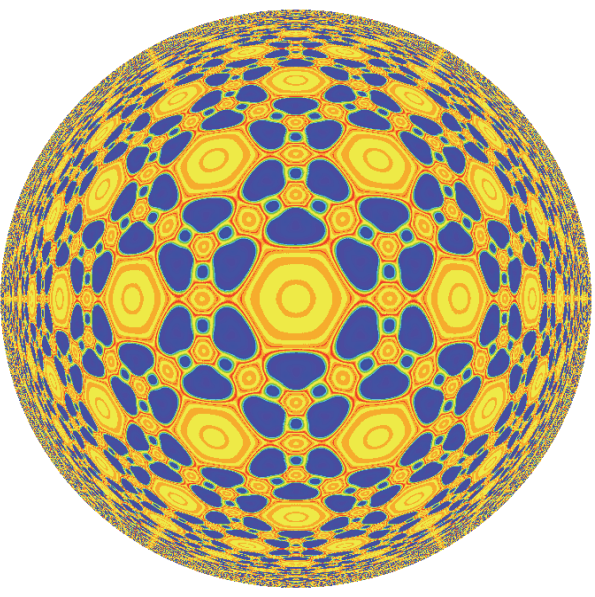

(b)

FIGURE 4: Colorful spherical wallpaper patterns with the $p 4 m$ (a) and $p 6 m$ (b) symmetry.

periods of the wallpaper pattern. However, if plane $Z=F$ is too far away from coordinate plane $X O Y$, the wallpaper pattern on the sphere may appear small so that we cannot identify symmetries of the wallpaper pattern well. Figure 3 illustrates the contrast effect of the setting of plane $Z=F$.

Given a wallpaper pattern, by central projection $\tau$, we can map it onto the sphere $S^{2}$ and obtain a corresponding spherical wallpaper pattern. We next explain how to implement it in more detail.

Suppose that $(a, b, c) \in R^{3}$ and $H_{f_{L}, w}(x)$ is an invariant mapping compatible with the symmetry of wallpaper group $w$. First, by central projection $\tau$, we obtain a corresponding point $((A / c) a,(A / c) b, F)$ on the projection plane $Z=F$. Second, let $H_{f_{L}, w}(x)$ be iteration function and let $x((A / c) a,(A / c) b)$ be initial point; using the color scheme of orbit trap, we assign a color to point $((A / c) a,(A / c) b, F)$. Finally, repeat the second step; by coloring unit sphere $S^{2}$ pointwise, we obtain a spherical pattern of the wallpaper group $w$ symmetry.
Figures 3-7 are ten patterns obtained by this manner. Except for Figure 3(b) in which the projection plane was set as $Z=-4 \pi$, all the other projection planes were set as $Z=-2 \pi$. We utilized the wallpaper patterns of Figure 1 to produce spherical patterns shown in Figure 4. For beauty, all the camera views are perpendicular to plane $X O Y$ and pass the origin, except for Figure 7(b), where the camera view aims at the equator of $S^{2}$. To better understand the effect of central projection, Figure 7 demonstrates spherical patterns that are observed from different perspectives.

\section{Additional Points}

The artistic patterns created in this article have significant aesthetic and economic value. We plan to produce some material objects with the help of simulation and printing technologies. We produced Figures $1-7$ in the $\mathrm{VC}++6.0$ programming environment with the aid of OpenGL, a powerful graphics software package. 


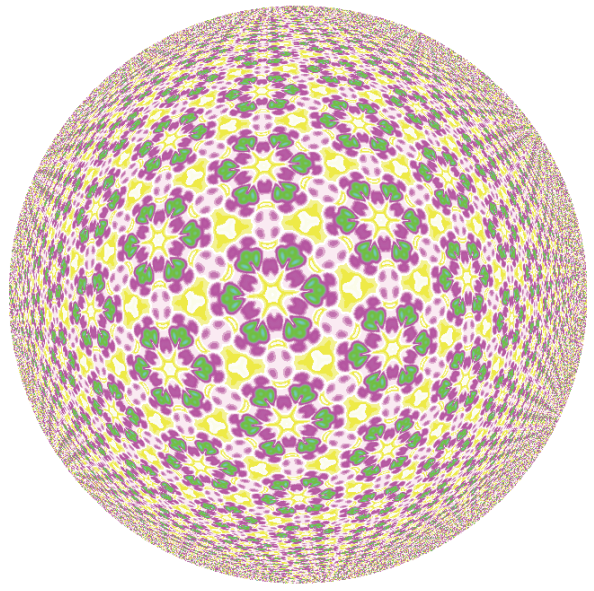

(a)

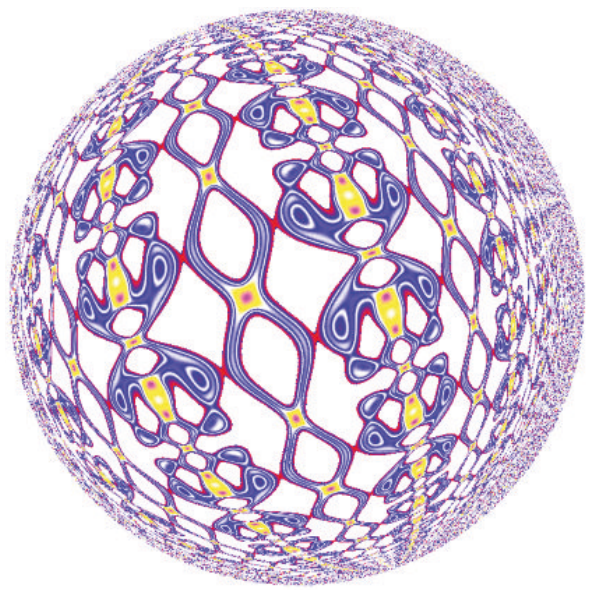

(b)

FiguRE 5: Colorful spherical wallpaper patterns with the $p 6 m$ (a) and pmm (b) symmetry.

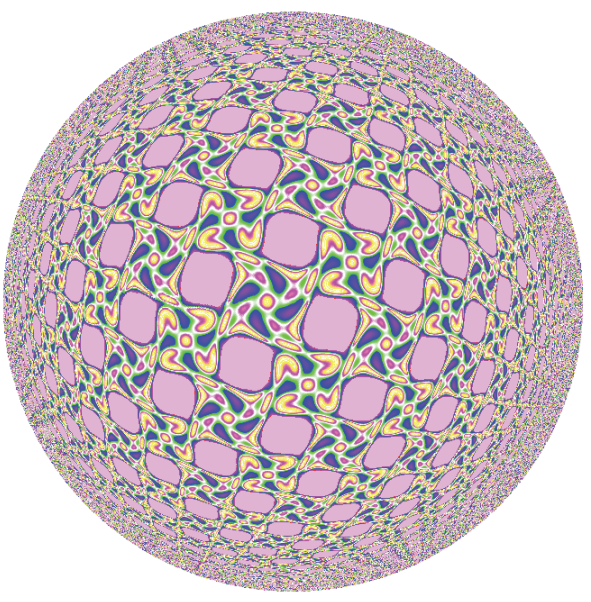

(a)

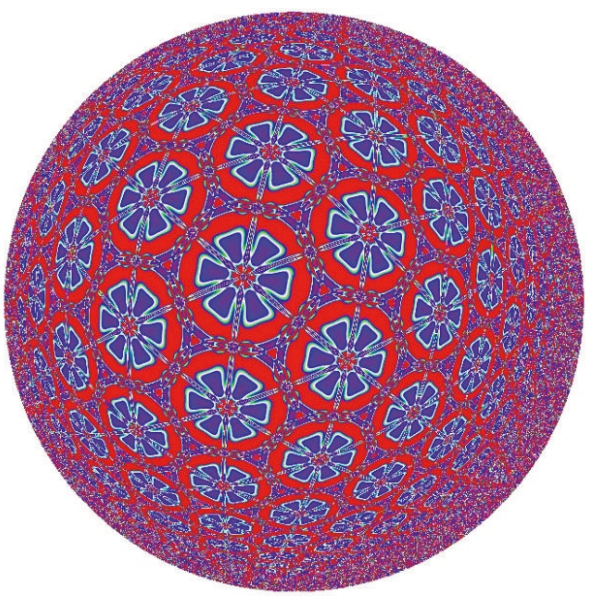

(b)

FIGURE 6: Colorful spherical wallpaper patterns with the $p 4$ (a) and $p 6 m$ (b) symmetry.

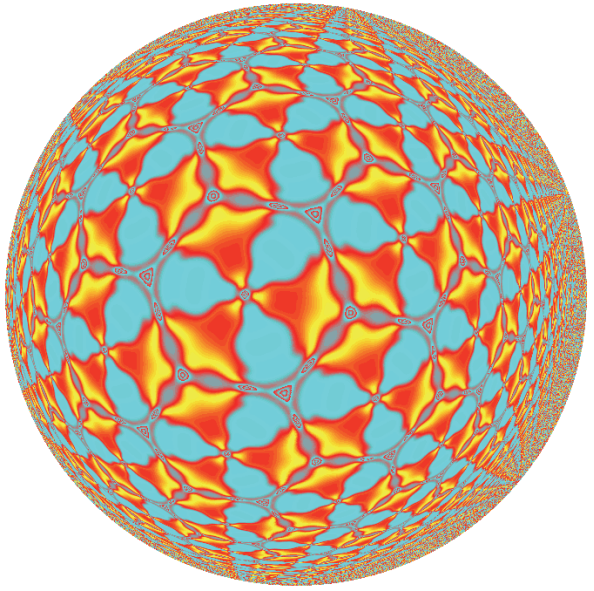

(a)

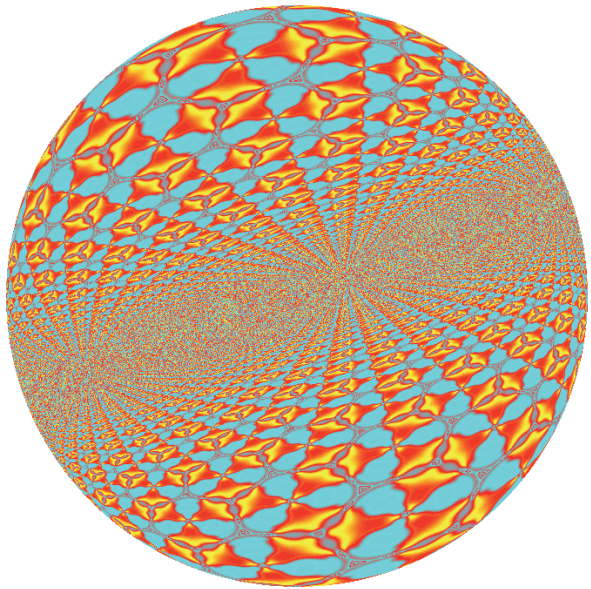

(b)

FIGURE 7: Two spherical wallpaper patterns with the $p 3 m$ symmetry. The camera view of (a) is perpendicular to plane XOY and passed the origin, while (b) aims at equator. 


\section{Conflicts of Interest}

The authors declare that there are no conflicts of interest regarding the publication of this paper.

\section{Acknowledgments}

The authors acknowledge Adobe and Microsoft for their friendly technical support. This work was supported by the National Natural Science Foundation of China (nos. 11461035, 11761039, and 61363014), Young Scientist Training Program of Jiangxi Province (20153BCB23003), Science and Technology Plan Project of Jiangxi Provincial Education Department (no. GJJ160749), and Doctoral Startup Fund of Jinggangshan University (no. JZB1303).

\section{References}

[1] E. Fedorov, "Symmetry in the plane," Proceedings of the Imperial St. Petersburg Mineralogical Society, vol. 2, pp. 245-291, 1891 (Russian).

[2] G. Pólya, "XII. Über die analogie der kristallsymmetrie in der ebene," Zeitschrift für Kristallographie-Crystalline Materials, vol. 60 , no. 1-6, 1924.

[3] J. Owen, The Grammar of Ornament, Van Nostrand Reinhold, 1910.

[4] P. S. Stevens, Handbook of Regular Patterns, MIT Press, London, UK, 1981.

[5] B. Grünbaum and G. C. Shephard, Tilings and Patterns, Cambridge University Press, Cambridge, UK, 1987.

[6] M. C. Escher, K. Ford, and J. W. Vermeulen, Escher on Escher: Exploring the Infinity, N. Harry, Ed., Abrams, New York, NY, USA, 1989.

[7] M. Field and M. Golubitsky, Symmetry in Chaos, Oxford University Press, Oxford, UK, 1992.

[8] N. C. Carter, R. L. Eagles, S. . Grimes, A. C. Hahn, and C. A. Reiter, "Chaotic attractors with discrete planar symmetries," Chaos, Solitons \& Fractals, vol. 9, no. 12, pp. 2031-2054, 1998.

[9] K. W. Chung and H. S. Y. Chan, "Symmetrical patterns from dynamics," Computer Graphics Forum, vol. 12, no. 1, pp. 33-40, 1993.

[10] J. Lu, Z. Ye, Y. Zou, and R. Ye, “Orbit trap rendering methods for generating artistic images with crystallographic symmetries," Computers and Graphics, vol. 29, no. 5, pp. 794-801, 2005.

[11] D. Douglas and S. John, "The art of random fractals," in Proceedings of Bridges 2014: Mathematics, Music, Art, Architecture, Culture, pp. 79-86, 2014.

[12] J. L. Alperin, Groups and Symmetry. Mathematics Today Twelve Informal Essays, Springer, New York, NY, USA, 1978.

[13] H. S. M. Coxeter and W. O. J. Moser, Generators and Relations for Discrete Groups, Springer, New York, NY, USA, 1965.

[14] I. R. Shafarevich and A. O. Remizov, "Linear algebra and geometry," in Gordon and Breach Science PUB, Springer, New York, NY, USA, 1981.

[15] J. H. Conway and N. J. A. Sloane, Sphere Packings, Lattices and groups, Springer, New York, NY, USA, 1993.

[16] T. Hahn, International Tables for Crystallography, Published for the International Union of Crystallography, Kluwer Academic Publishers, 1987.
[17] V. E. Armstrong, Groups and Symmetry, Springer, New York, NY, USA, 1987.

[18] K. W. Chung and H. M. Ma, "Automatic generation of aesthetic patterns on fractal tilings by means of dynamical systems," Chaos, Solitons \& Fractals, vol. 24, no. 4, pp. 1145-1158, 2005.

[19] P. Ouyang and X. Wang, "Beautiful math-aesthetic patterns based on logarithmic spirals," IEEE Computer Graphics and Applications, vol. 33, no. 6, pp. 21-23, 2013.

[20] P. Ouyang, D. Cheng, Y. Cao, and X. Zhan, “The visualization of hyperbolic patterns from invariant mapping method," Computers and Graphics, vol. 36, no. 2, pp. 92-100, 2012.

[21] P. Ouyang and R. W. Fathauer, "Beautiful math, part 2: aesthetic patterns based on fractal tilings," IEEE Computer Graphics and Applications, vol. 34, no. 1, pp. 68-76, 2014.

[22] P. Ouyang and K. Chung, "Beautiful math, part 3: hyperbolic aesthetic patterns based on conformal mappings," IEEE Computer Graphics and Applications, vol. 34, no. 2, pp. 72-79, 2014.

[23] P. Ouyang, L. Wang, T. Yu, and X. Huang, "Aesthetic patterns with symmetries of the regular polyhedron," Symmetry, vol. 9, no. 2 , article no. $21,2017$. 


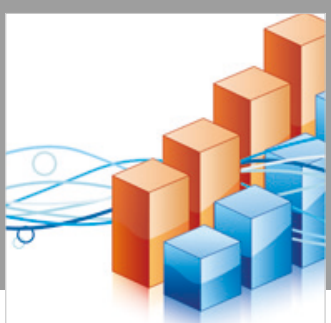

Advances in

Operations Research

\section{-n-m}
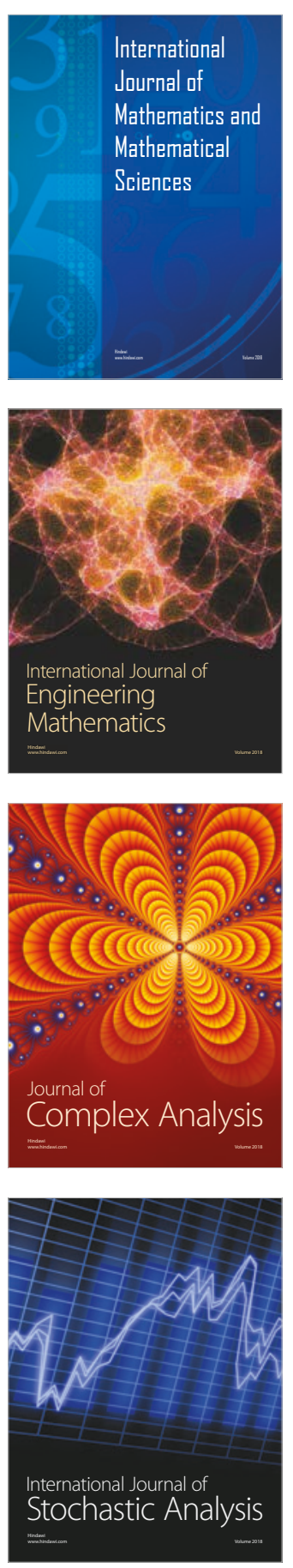
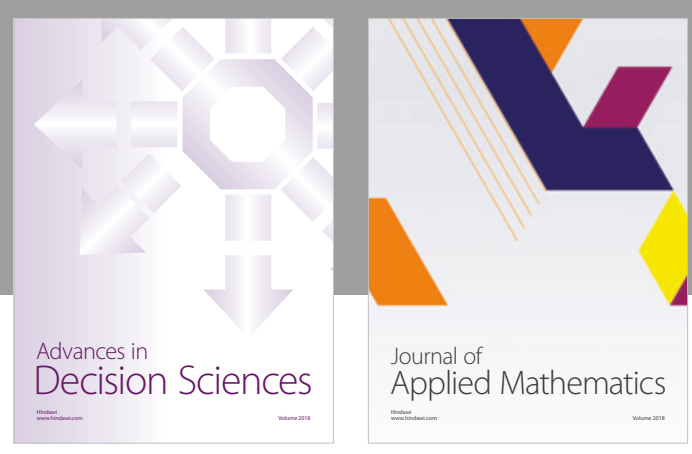

Journal of

Applied Mathematics
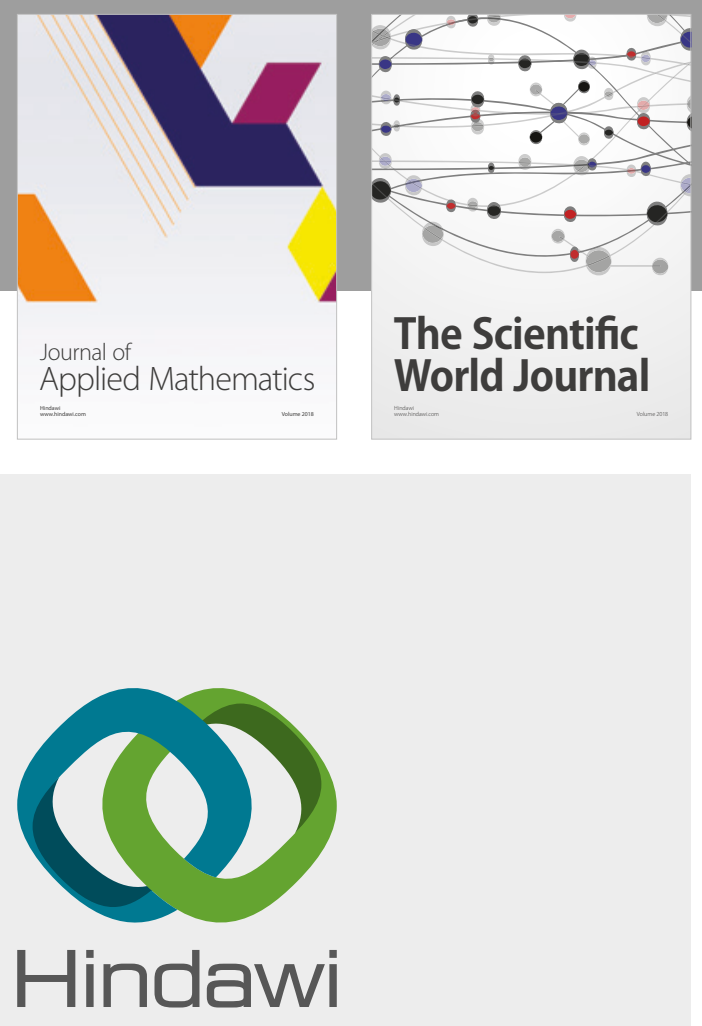

Submit your manuscripts at

www.hindawi.com

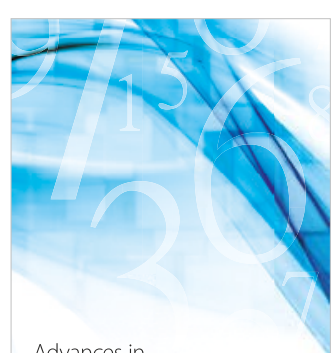

Advances in
Numerical Analysis
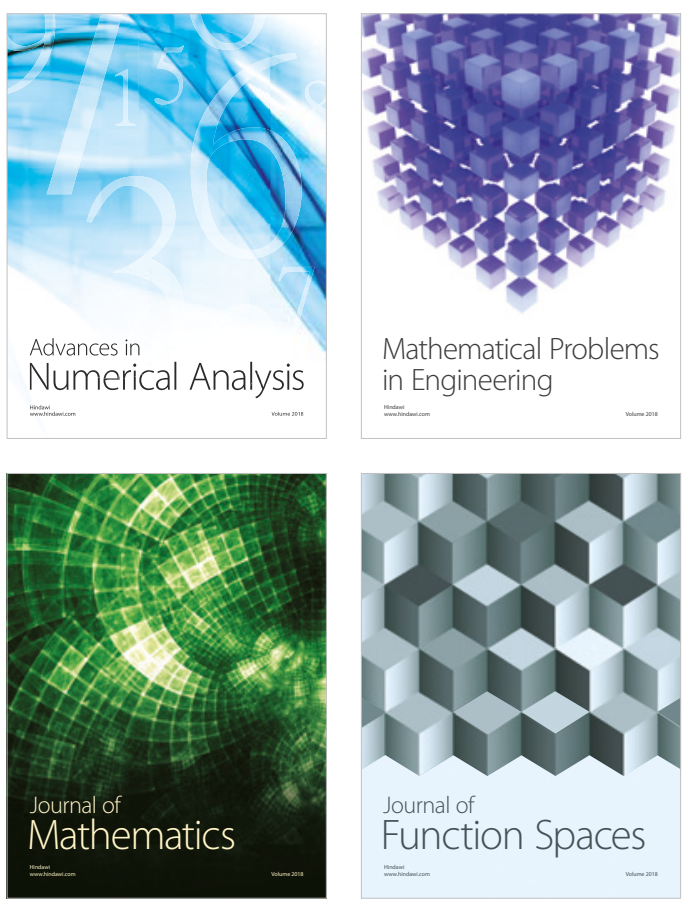

Mathematical Problems in Engineering

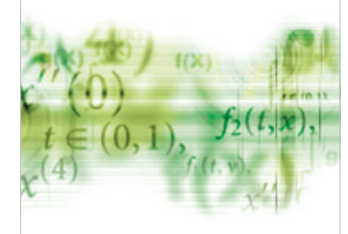

International Journal of

Differential Equations

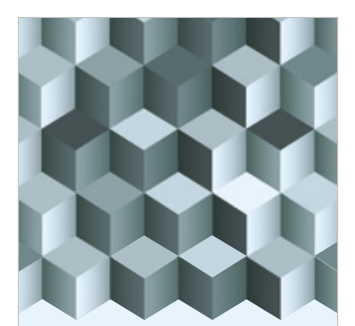

Journal of

Function Spaces
The Scientific

World Journal

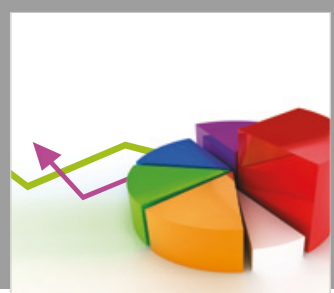

Journal of

Probability and Statistics
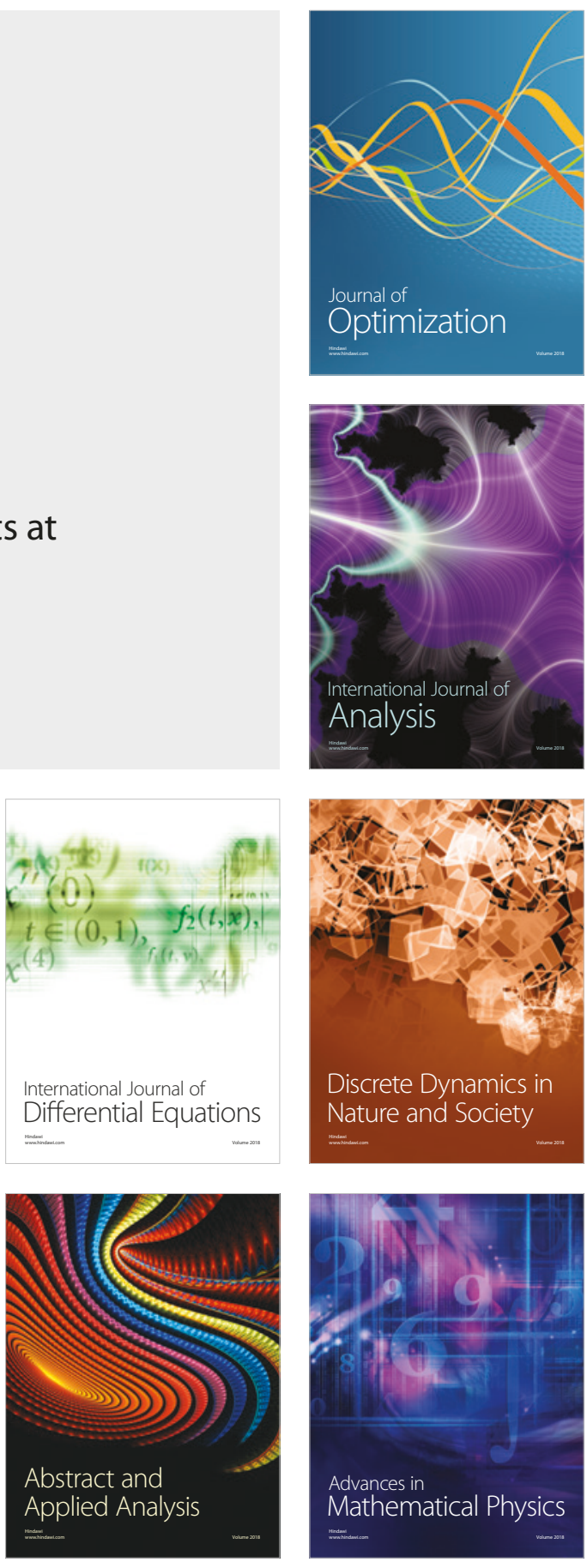